\title{
Is the 3-D magnetic null point with a convective electric field an efficient particle accelerator?
}

\author{
J.-N. Guo ${ }^{1,2,3}$, J. Büchner ${ }^{1}$, A. Otto ${ }^{4}$, J. Santos ${ }^{1}$, E. Marsch ${ }^{1}$, and W.-Q. Gan ${ }^{2}$ \\ 1 Max-Planck-Institut für Sonnensystemforschung, Katlenburg-Lindau, Germany \\ 2 Purple Mountain Observatory, Chinese Academy of Sciences, Nanjing, PR China \\ 3 University of Glasgow, Glasgow, UK \\ e-mail: j.guo@astro.gla.ac.uk \\ 4 Geophysical Institute, University of Alaska Fairbanks, USA
}

Received 18 September 2009 / Accepted 23 January 2010

\section{ABSTRACT}

\begin{abstract}
Aims. We study the particle acceleration at a magnetic null point in the solar corona, considering self-consistent magnetic fields, plasma flows and the corresponding convective electric fields.

Methods. We calculate the electromagnetic fields by 3-D magnetohydrodynamic (MHD) simulations and expose charged particles to these fields within a full-orbit relativistic test-particle approach. In the 3-D MHD simulation part, the initial magnetic field configuration is set to be a potential field obtained by extrapolation from an analytic quadrupolar photospheric magnetic field with a typically observed magnitude. The configuration is chosen so that the resulting coronal magnetic field contains a null. Driven by photospheric plasma motion, the MHD simulation reveals the coronal plasma motion and the self-consistent electric and magnetic fields. In a subsequent test particle experiment the particle energies and orbits (determined by the forces exerted by the convective electric field and the magnetic field around the null) are calculated in time.

Results. Test particle calculations show that protons can be accelerated up to $30 \mathrm{keV}$ near the null if the local plasma flow velocity is of the order of $1000 \mathrm{~km} \mathrm{~s}^{-1}$ (in solar active regions). The final parallel velocity is much higher than the perpendicular velocity so that accelerated particles escape from the null along the magnetic field lines. Stronger convection electric field during big flare explosions can accelerate protons up to $2 \mathrm{MeV}$ and electrons to $3 \mathrm{keV}$. Higher initial velocities can help most protons to be strongly accelerated, but a few protons also run the risk to be decelerated.

Conclusions. Through its convective electric field and due to magnetic nonuniform drifts and de-magnetization process, the 3-D null can act as an effective accelerator for protons but not for electrons. Protons are more easily de-magnetized and accelerated than electrons because of their larger Larmor radii. Notice that macroscopic MHD simulations are blind to microscopic magnetic structures where more non-adiabatic processes might be taking place. In the real solar corona, we expect that particles could have a higher probability to experience a de-magnetization process and get accelerated. To trigger a significant acceleration of electrons and even higher energetic protons, however, the existence of a resistive electric field mainly parallel to the magnetic field is required. A physically reasonable resistivity model included in resistive MHD simulations is direly needed for the further investigations of electron acceleration by parallel electric fields.
\end{abstract}

Key words. acceleration of particles - magnetohydrodynamic (MHD) - magnetic fields - Sun: flares

\section{Introduction}

Large solar flares are very powerful explosions near the solar surface, releasing up to $10^{32}-10^{33}$ erg in $10^{2}-10^{3} \mathrm{~s}$ into plasma bulk flow energy, thermal and non-thermal energy. Accelerated electrons and ions are estimated to carry $10 \%$ to $50 \%$ of the total magnetic energy released in flares (Lin \& Hudson 1976). These particles are assumed to be accelerated by a number of mechanisms occurring at different stages and locations of a flare evolution. Theoretically, a number of acceleration mechanisms has been considered, e.g. (1) direct current (DC) field acceleration in strong electric fields generated in current-carrying loops or in current sheets in the corona (Litvinenko 1996); (2) stochastic acceleration by MHD turbulence where particle energy is gained or lost, e.g. in fast plasma reconnection outflows; (3) shock acceleration by a fast termination shock involved in an inhomogeneous boundary. For a review of acceleration mechanisms, see e.g. Aschwanden (2002).
Recent solar observations gave important clues about the nature of particle acceleration on the Sun. According to flare models derived from these observations, the hard X-ray (HXR) and $\gamma$-ray emissions at the chromospheric footpoints of magnetic loops are supposed to be produced by bremsstrahlung that is related to high-energy electrons and protons transported downward along flare loops from acceleration sites higher up in the corona. One can deduce the particle energy spectrum from the observed HXR and $\gamma$-ray spectrum, considering either thicktarget models (where the injected particles lose most of their energy in Coulomb collisions with dense, ambient plasma and emit hard X-ray) or thin-target models (where electrons continuously propagate without being braked, and the X-ray spectrum is nearly unchanged from the injection spectrum) (Brown 1971). The appearance of another coronal HXR source on top of the soft-X-ray loops (Masuda et al. 1994) is considered to be an indication of the first energy-release process by accelerated particles encountering the flare loop. The location of the acceleration site has also been estimated by calculating the time-of-flight distance 
(Aschwanden et al. 1996) from observations. However, the inversion of photon spectra to particle spectra could not answer the question how particles are accelerated in the corona and then transported downward. It is very difficult to infer from observations how the different acceleration mechanisms work together, and how large-scale fields, which accelerate particles, can build up. Hence numerical calculations are necessary to study the microscopic acceleration process in macroscopic configurations.

Magnetic reconnection plays an important role in building up large scale electric fields able to accelerate particles. In the past decades, a substantial amount of work was carried out to investigate the acceleration of test particles in reconnection electromagnetic fields. Three different approaches were taken to model the magnetic and electric fields:

Early researchers often took an analytical prescription of the 2D X-type reconnecting magnetic field, imposing a constant and uniform electric field in the third dimension (e.g. Speiser 1965, 1967; Bruhwiler \& Zweibel 1992; Mori et al. 1998; Browning \& Vekstein 2001; Zharkova \& Gordovskyy 2004; Efthymiopoulos et al. 2005; Hannah \& Fletcher 2006). The conclusion from these test particle calculations is that with larger electric fields and with additional guiding magnetic fields in the current sheet, particles can be accelerated more efficiently. A longitudinal (guide) magnetic field magnetizes the charged particles and reduces their probability of being ejected from the current sheet so that particles can gain more energy from the electric field (Litvinenko 1996). A power-law distribution of accelerated particles is usually obtained and used for comparison with observations. Nevertheless, the reconnection process itself, which produces the electric field, is neglected and the magnetic and electric fields are prescribed and independent. Therefore these simulations provide a qualitative analysis of the acceleration rather than quantitative results for particle energies and spectrum.

A further step is to apply analytic reconnection solutions for test particles so as to set up self-consistent electric and magnetic fields. Based on simplified Ohm's law and Ampere's law, the electric fields are calculated from the analytic magnetic fields, plasma velocities and sometimes also from resistivity models (e.g. Sakai 1992; Heerikhuisen et al. 2002; Craig \& Litvinenko 2002; Wood \& Neukirch 2005; Hamilton et al. 2005; Dalla \& Browning 2005, 2008). In these investigations, the reconnection fields are not independent free parameters, but are obtained from MHD solutions. This approach results in a more reliable prediction of the properties of energetic particle populations. For example, Dalla and Browning (e.g. Dalla \& Browning 2005, 2008) used an analytical model of magnetic and electric fields for kinematically prescribed ideal plasma flows around a potential 3D null (Priest \& Titov 1996). They studied particle acceleration for spine and fan reconnection. For the same configuration of the magnetic field, different plasma flows correspond to different convection electric fields. In the spine reconnection case, plasma flows in and out through the center of the null, resulting in an azimuthal electric field. An efficient acceleration is obtained assuming that the electric field could be as large as $1 \mathrm{kV} / \mathrm{m}$. In the fan reconnection case, the plasma flow has another azimuthal component and the acceleration is less efficient, because fewer particles can reach the regions of strong electric fields. Note however that the analytic solution of the electric field contains a singularity at the center $(E \rightarrow \infty$ at $R=0)$, which leads to infinite runaway acceleration. Also, lack of information on the actual scale and magnitude of the reconnecting magnetic field as well as the strength and configuration of the real plasma flows hinders the process of obtaining realistic acceleration energies and spectrum.

A third possible approach is to use the output of selfconsistent MHD simulations. Test particles can be traced in the electromagnetic fields obtained e.g. by ideal or resistive MHD numerical simulations (e.g. Schopper et al. 1999; Dmitruk et al. 2003; Turkmani et al. 2006; Liu et al. 2009; Karlicky \& Barta 2006). This combination of the test particle method with MHD simulations can provide a semi-realistic and consistent field geometry and strength for the charged particles. Note that because of the coarse resolution of the simulated MHD fields, the magnetic and electric fields have to be interpolated for test particle calculations. Furthermore, a resistivity model has to be carefully used in MHD simulations, as the macroscopic MHD does not consider the microphysical effects that control the resistivity.

The direct way to gain energy without any interference of the perpendicular gyromotion is acceleration by a parallel electric field, because magnetized particles can move freely in the direction parallel to the magnetic field. A simple model as mentioned before is the direct acceleration inside an electric current sheet on which a parallel guiding magnetic field is superposed. Nevertheless, the value of the uniform electric field and the guide magnetic field as well as the width of current sheet are prescribed without sufficient support by observations or simulations. For an electron to be accelerated to $100 \mathrm{keV}$ in a sub-Dreicer electric field, where $E \approx 9 \times 10^{-6} \mathrm{~V} / \mathrm{m}$, an unrealistically long current sheet of more than $10^{7} \mathrm{~m}$ in length would be needed. To reach the same energy in a super-Dreicer field, the length can be much shorter, i.e. $10^{2} \mathrm{~m}$, but then the electric field is unrealistically large (up to $10^{3} \mathrm{~V} / \mathrm{m}$ ) (Aschwanden 2002). A more complicated $3 \mathrm{D}$ model of parallel electric fields can be obtained by resistive MHD simulations. According to Ohm's law, parallel electric fields are balanced as a resistive electric field $\boldsymbol{E}_{\mathrm{res}}=\eta \boldsymbol{j}$. So one would qualitatively expect that a parallel current density $j_{\|}$and the existence of a diffusion region with a considerable resistivity $\eta$ would favour direct acceleration. Nevertheless, any quantitative results based on prescribed $\eta$ are somehow arbitrary, since there is no generic way to to parameterize the non-ideal property of the collisionless corona plasma in MHD simulation. For example, test particle acceleration to energies up to $100 \mathrm{GeV}$ (Turkmani et al. 2006) might be a result of using a numerical "hyper-resistivity", which stabilizes the MHD code. Also, the parallel electric fields obtained from kinetic processes are in general confined to regions on the ion inertia scale (Hesse et al. 1999), much smaller than the macroscopic MHD grid scales, which can therefore affect and accelerate only a few particles.

On the other hand it might be possible to accelerate particles in a perpendicular convective electric field (which is much larger than resistive electric fields in big Reynolds-number plasmas) due to drift forces and non-adiabatic motion. The mechanism of this acceleration will be further described in Sect. 3. A null point is supposed to be the most probable location to switch on this convective acceleration. Recent observations (e.g. Aulanier et al. 2000; Fletcher et al. 2001; Des Jardins et al. 2009) indicate that 3-D null points are likely to be common in solar corona configurations. Unfortunately, there is no direct observation of the magnitude of the magnetic and electric fields around coronal nulls. One can obtain the magnetic field around a 3-D null point in the corona however by extrapolating typical photospheric fields. A parallel electric field is undoubtedly effective for acceleration. Still, its real strength is quite unknown, because in resistive MHD models it is controlled by ad-hoc prescribed or numerical resistivity. We will therefore concentrate on perpendicular electric fields due to convective electric fields (which are 
determined by convective plasma flows) to explore the acceleration near a 3-D null point.

The paper is organized as follows. We first describe the codes for MHD simulation and the methods of test-particle calculation. For a 3-D null configuration we analyze theoretically the process of particle acceleration by convective electric fields due to non-adiabatic and drift motion in the non-uniform magnetic fields near the null. Thereafter, we calculate the test-particle energy gains and orbits around the null for different electric fields (plasma flows). We also test different initial distributions of the particles to investigate their acceleration under different initial conditions. We conclude that the convective electric field near the 3-D null point could work as an effective accelerator for protons under realistic assumptions for the field strength and plasma flow velocities. To efficiently accelerate electrons though it appears to be necessary to include parallel electric fields, because electrons are hardly de-magnetized and only shortly drifting in the direction of the convective electric field.

\section{The field structure - MHD simulation results}

A three-dimensional cartesian MHD model was used to describe the evolution of the large-scale magnetic and electric fields from the solar photosphere to the lower corona (e.g. Büchner et al. 2004; Büchner 2007). This MHD model solves the following equations, including the continuity Eq. (1), momentum Eq. (2), induction Eqs. (3) and energy Eq. (4), together with Ohm's law (5), Ampere's law (6) and an equation of state (7):

$$
\begin{aligned}
& \frac{\partial \rho}{\partial t}=-\nabla \cdot \rho \boldsymbol{u} \\
& \frac{\partial \rho \boldsymbol{u}}{\partial t}=-\nabla \cdot\left[\rho \boldsymbol{u} \boldsymbol{u}+\frac{1}{2}\left(p+B^{2}\right) I-\boldsymbol{B} \boldsymbol{B}\right]-v \rho\left(\boldsymbol{u}-\boldsymbol{u}_{n}\right) \\
& \frac{\partial \boldsymbol{B}}{\partial t}=-\nabla \times \boldsymbol{E}=\nabla \times(\boldsymbol{u} \times \boldsymbol{B}-\eta \boldsymbol{j}) \\
& \frac{\partial p}{\partial t}=-\nabla \cdot p \boldsymbol{u}-(\gamma-1) p \nabla \cdot \boldsymbol{u}+2(\gamma-1) \eta \boldsymbol{j}^{2} \\
& \boldsymbol{E}=-\boldsymbol{u} \times \boldsymbol{B}+\eta \boldsymbol{j} \\
& \nabla \times \boldsymbol{B}=\boldsymbol{j} \\
& p=2 n k_{B} T .
\end{aligned}
$$

The variables in Eqs. ((1)-(7)) are normalized (dimensionless) by dividing by the normalization quantities. The normalization magnetic field, electric field and plasma velocity have the relationship: $E_{0}=B_{0} u_{0}$. Typical values are $B_{0}=1 \mathrm{G}, u_{0}=$ $50 \mathrm{~km} \mathrm{~s}^{-1}$ and $E_{0}=5 \mathrm{~V} / \mathrm{m}$. To simulate the global magnetic field from the bottom of the photosphere up to the corona, the normalization length scale is $L_{0}=500 \mathrm{~km}$. The total box length in the horizontal $x$ and $y$ direction is $93 L_{0}(46.5 \mathrm{Mm})$, and in the vertical $z$ direction it is near $31 L_{0}(15.5 \mathrm{Mm})$. The height of the transition region is about $5 L_{0} \sim 2.5 \mathrm{Mm}$.

Our initial 3-D magnetic field configuration was obtained by potential-field extrapolation (Otto et al. 2007) of a quadrupolar analytic magnetic field (see Fig. 2 for images and Appendix A for equations). The magnitude of the magnetic field decreases with height. Its maximum value $\left(293 B_{0}\right)$ is located at the bottom of the box. It drops to about $150 B_{0}$ at the transition layer and to just tens of $B_{0}$ in the corona. In such a configuration, which should not be too rare in the solar atmosphere, the magnetic field

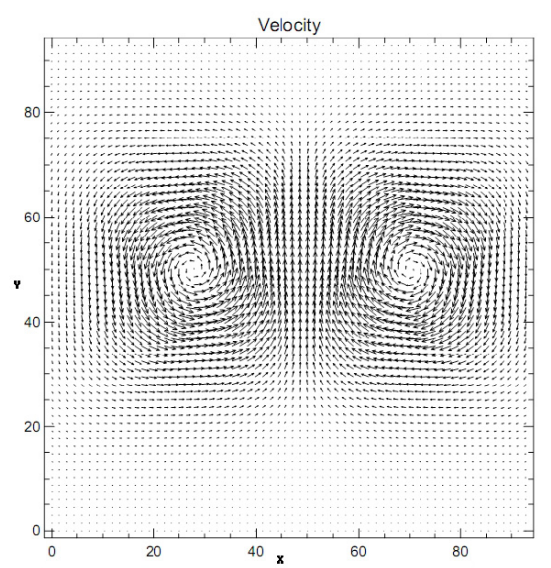

Fig. 1. Rotating neutral gas velocity imposed at the bottom of the simulation box.

cancels near the central vertical line of the box. Since the magnetic field vanishes, a null point is located near the box center. The approximate location of the magnetic null point being 46.4, $46.4,14.6$ is determined by the minimum of the magnetic field magnitude which is $0.08 \mathrm{G}$ on the MHD grids. This reference point is not the location of the actual null point (which requires an interpolation of the magnetic fields between the grid points), but is in its immediate vicinity. For simplification, we call this reference point "the null" throughout the paper. This field configuration was our starting point for an investigation of particle acceleration near a 3-D null point.

The boundary conditions were defined so that the MHD equations remained invariant under transformation of the MHD variables (Otto et al. 2007). The top boundary (at $z=L_{z}$ ) was an open boundary. The four lateral boundary conditions (at $x=0, x=L_{x}, y=0, y=L_{y}$ ) were line-mirroring symmetric. The bottom boundary condition (at $z=0$ ) for the magnetic field was obtained by considering that the field should satisfy $\nabla \cdot \boldsymbol{B}=0$ and that there should be no horizontal currents $\left(j_{x}=(\nabla \times \boldsymbol{B})_{x}=0\right.$, and $\left.j_{y}=(\nabla \times \boldsymbol{B})_{y}=0\right)$ in the photosphere at $z=0$. For the plasma motion, the momentum flux through the bottom was set to be zero $\left(u_{z}(z=0)=0\right)$, meaning no emerging flux in the photosphere. Since the horizontal plasma motion in the solar photosphere plays an important role in the build-up of electric currents in solar atmosphere (e.g. Santos \& Büchner 2007; Büchner 2006), we applied two vortices of neutral gas motion at the bottom with an average speed of about $0.0137 u_{0}$ (see Fig. 1). The last term on the right-hand side of the momentum equation (Eq. (2)) represents the transfer of momentum from the neutral gas to the plasma through collisions. The neutral gas velocity was set to be a horizontal vortex with $\boldsymbol{u}_{n}=\nabla \times\left(U \boldsymbol{e}_{z}\right)$, where $U$ is a scalar potential so as to keep $\nabla \cdot \boldsymbol{u}_{\boldsymbol{n}}=0$ to inhibit the piling up of the plasma and magnetic field. The plasma is dragged behind the neutral gas through collisional interaction with the gas. The collision frequency $v$ is height dependent, attaining its maximum at the bottom and decreasing exponentially with height. Therefore the plasma motion is coupled with the neutral gas in the photosphere and chromosphere, while it is decoupled from the neutral gas motion in the corona. Hence the whole evolution of an initially relaxed equilibrium state is due to plasma flows induced by the moving photospheric neutral gas.

The complete set of Eqs. ((1)-(7)) was numerically solved in a 3-D cartesian box with finite-difference discretization methods. The grid was chosen to be equidistant in the $x$ and $y$ directions, both with 128 grid points and $\Delta_{x}=\Delta_{y}=0.73 L_{0}$. It is 

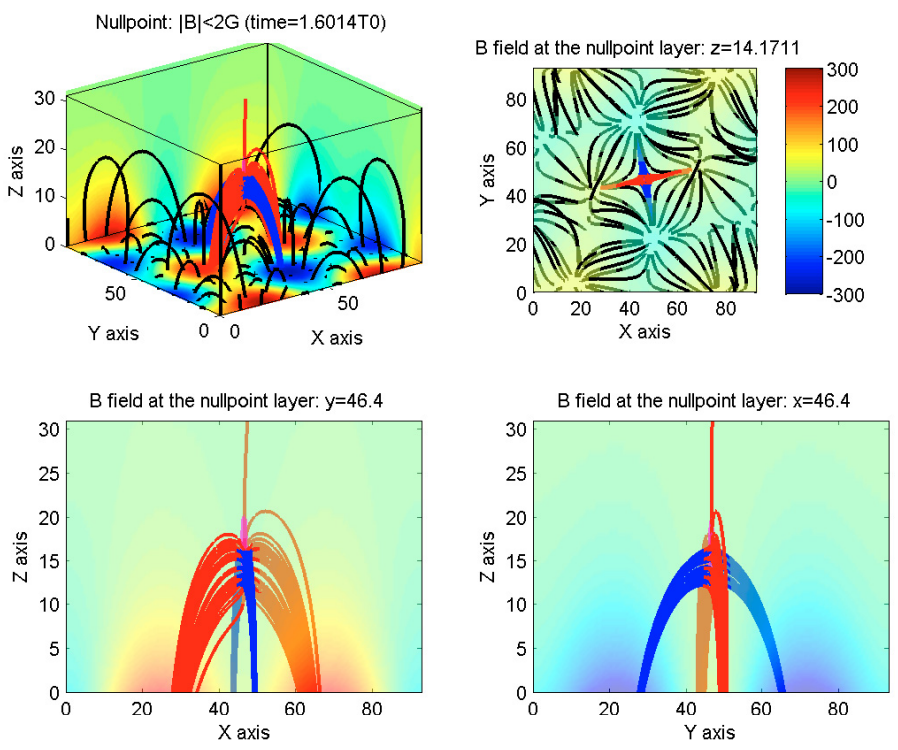

Fig. 2. Magnetic field configuration with a null in the center of the box. The upper-left figure shows the 3-D view of the whole simulation box extending from the photosphere to the corona. The upper-right figure is a $x-y$ face-on view of the null at $z=14.17$. The bottom-left/bottomright figure is the $x-z / y-z$ cut through the center of the $y$-axis/ $x$-axis, where the null is located. The black lines are the global magnetic field lines. The blue lines are the magnetic field lines leaving from the weak field region $(B<2)$, while the red lines are the magnetic flied lines coming into the region.

non-equidistant in the $z$ direction (with 64 grid points): the resolution decreases with height. The grid size is $\Delta_{z}=0.3 L_{0}$ at the bottom and stretched to $\Delta_{z}=1.0 L_{0}$ at the top. Equations (1) to (4) were advanced in time with a second-order accurate leapfrog scheme (Potter 1973), because it has a very low numerical dissipation. A Lax scheme was used in the first and last step.

The location of the minimum numerical magnetic field slightly shifts in height from $(46.4,46.4,14.6)$ to $(46.4,46.4$, 14.2) after the simulation of 1.6 Alfvén times and its value becomes $(0.0484 \mathrm{G})$. The weak field region $(B<2 \mathrm{G})$ in Fig. 2 extends between $x: 45.0-47.1, y: 45.0-47.1$, and $z: 12.0-20.5$. We calculated the Jacobian matrix $M_{i j}=\partial B_{i} / \partial x_{j}$ to evaluate the structure (Lau \& Finn 1990) around the null. The eigenvalues of the matrix at the null point are about $(2.1,-1.7,-0.3)$, following the category of a negative null. The first positive eigenvalue defines the spine, while the last negative ones indicate a fan surface. The eigenvector of the matrix gives the direction of a corresponding spine or fan plane: the spine path is in the direction of $0.18,-0.98,0.03$ (followed by the blue field lines in Fig. 4); the fan surface is a plane defined by two vectors $(-0.98,-0.18,-0.01)$ and $(0,0,1)$, i.e. a vertical plane crossing the center of the red field lines in the figure. Since the second eigenvalue $(-0.3)$ for the fan plane is much smaller compared to the first $(-1.7)$, the field lines tend to follow the direction of $-0.98,-0.18,-0.01$ (red lines in the horizontal direction) rather than the vertical direction 0, 0, 1 (Restante et al. 2009). Actually, if the last eigenvalue vanishes, the $3 \mathrm{D}$ null point simply equals 2D X-point where no field lines extend in the vertical direction. These structures can also be seen in Figs. 6 and 7, where magnetic fields are shown as black lines.

Driven by the horizontal photospheric motion, the plasma in the whole simulation box evolves. It reaches a state with an average bulk velocity of $0.42 u_{0}$ after the simulation (top image
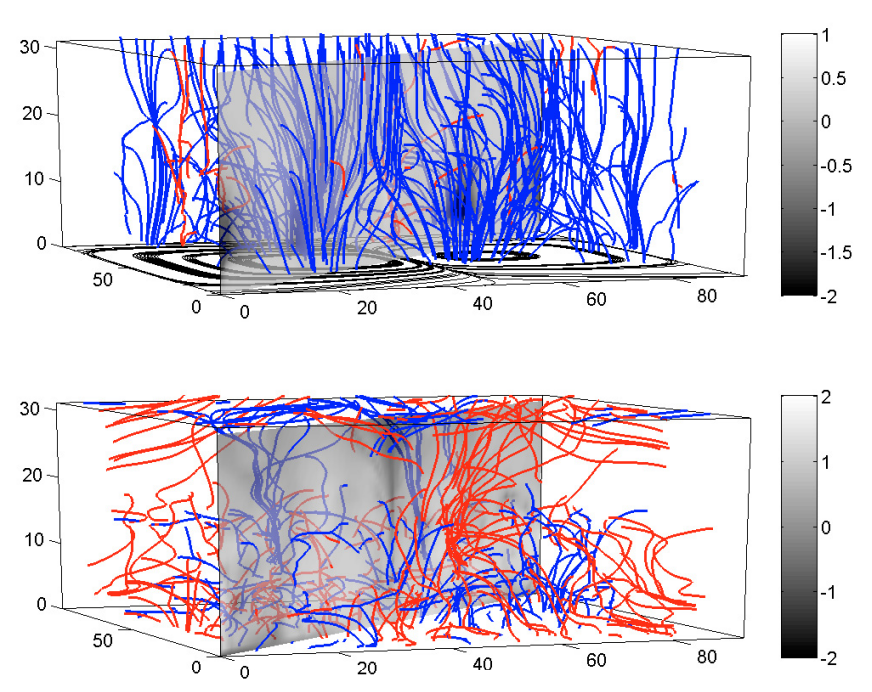

Fig. 3. Plasma velocity flow lines (top) and corresponding convective electric fields (bottom). The red lines represent velocity flow (electric field) lines with positive (upward directed) $u_{z}\left(E_{z}\right)$. The blue lines indicate negative (downward directed) $u_{z}\left(E_{z}\right)$. The diagonal cross-section plane in the top image depicts the grey-coded normalized value of $u_{z}$. The cross-section plane in the bottom image shows the grey-scale $\lg \left(E_{\text {con }} / E_{0}\right)$.

in Fig. 3). The global configuration and topology of the magnetic fields do not show any obvious change. Figure 2 shows the evolved global configuration of the magnetic fields with a magnetic null in the corona. However, convective electric fields arise in the whole box (bottom image in Fig. 3) due to the plasma motion across the magnetic field (top image in Fig. 3). Since both the magnetic field and plasma flows are stronger in the chromosphere, the convective electric field $-\boldsymbol{u} \times \boldsymbol{B}$ develops more strongly in the lower part of the box. The convective electric field has an average value of $4.08 E_{0}$ in the whole box, and its maximum value is $3333 E_{0}$ at $(50.03,68.15,2.10)$ in the chromosphere. Near the null, however, the convective electric field is only $0.0036 E_{0}$ (bottom image of Fig. 4), because the magnetic field is minimum $\left(0.05 B_{0}\right)$ and the plasma bulk velocity is $0.233 u_{0}$. Figure 4 shows the resulting plasma flow lines and the convective electric fields (as black lines) close to the null.

Notice that throughout the simulation box the resistive electric field $\eta \boldsymbol{j}$ is kept very small, because $\eta$ is chosen so that most of solar corona is considered as ideal plasma. Anomalous resistivity is switched on at places where the local current carrier velocity, defined as $\boldsymbol{V}_{\mathrm{cc}}=\boldsymbol{j} / n e$, exceeds the electron thermal speed, $V_{\text {the }}$, which indicates the onset of a fluid-type electrostatic Buneman instability (Büchner 2007). The switched-on effective resistivity was given by Büchner \& Elkina (2006) and reads

$\eta_{\mathrm{eff}}=\frac{0.01}{\varepsilon_{0} \omega_{\mathrm{pe}}} \frac{T_{\mathrm{e}}}{T_{\mathrm{i}}} \cdot F F \cdot \frac{V_{\mathrm{cc}}}{V_{\mathrm{the}}}$,

where $\omega_{\mathrm{pe}}=\sqrt{\left(n e^{2} / \varepsilon_{0} m_{\mathrm{e}}\right)}$ is the electron plasma frequency, $\varepsilon_{0}$ is the vacuum electric permeability, and $T_{\mathrm{i}}$ and $T_{\mathrm{e}}$ are the ion and electron temperatures considered to be equal for solar coronal conditions. $F F$ is a filling factor, which is introduced to bridge the gap between the MHD grid scale $(\sim 500 \mathrm{~km})$ and the dissipation scale, which is about the ion skin depth $\left(\sim c / \omega_{\mathrm{pi}} \sim 7 \mathrm{~m}\right.$ for $n_{\mathrm{p}}=10^{15} \mathrm{~m}^{-3}$ ).

The simulated resistive electric field has its maximum value of $0.08 E_{0}$ in the transition region at $(48.6,69.6,4.6)$. Around 
Plasma velocity (black lines) \& Mag. fields (blue: outward; red: inward)

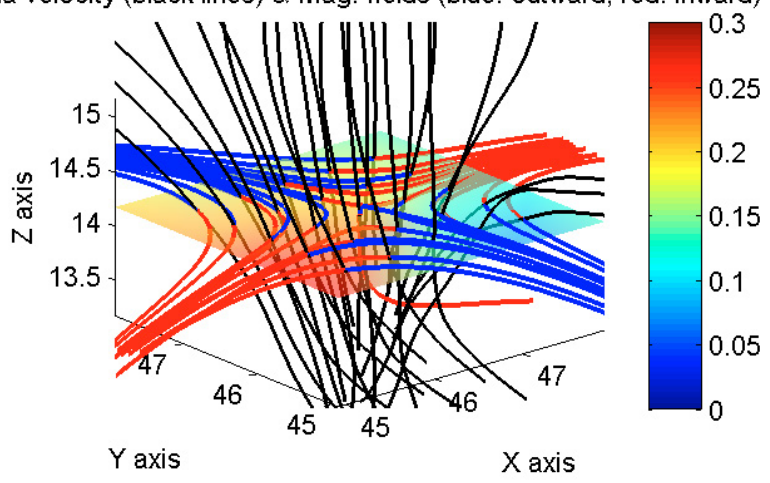

Convective fields (black lines) \& Mag. fields (blue: outward; red: inward)

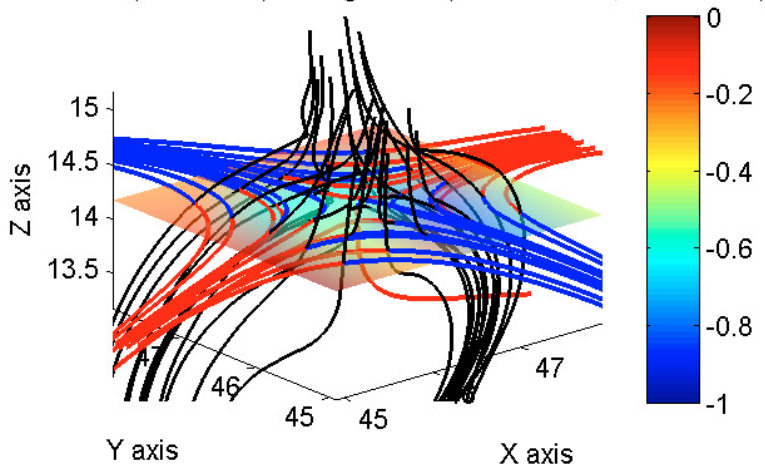

Fig. 4. Plasma velocity flow lines (top) and the corresponding convective electric fields (bottom) near the null. The red lines represent the magnetic fields coming into the weak field region $(B<2)$, while the blue ones are field lines leaving the region. The black lines indicate the plasma velocity flow lines (top) and convective electric field lines (bottom), with $u_{z}<0$ and $E_{z}<0$. The horizontal layers cutting through the null show the strength of the normalized plasma velocity $u(t o p)$ and the value of $\lg \left(E_{\mathrm{con}} / E_{0}\right)$ (bottom). The color-coding is indicated on the right side.

the null, no anomalous resistive electric field is switched on by the end of our simulation at $t=1.6 \tau_{A}$. We therefore considered only the perpendicular convective electric field and investigated the acceleration ability of the convective electric field around the 3-D null point.

\section{How can a convective electric field accelerate particles?}

The basic relativistic equation of motion of an individual charged particle in an electromagnetic field includes the acceleration by the electric field, $\boldsymbol{E}$, and the Lorentz force exerted by the magnetic field, $\boldsymbol{B}$ :

$\frac{\mathrm{d} m \gamma \boldsymbol{v}}{\mathrm{d} t}=q(\boldsymbol{E}+\boldsymbol{v} \times \boldsymbol{B})$,

$\frac{\mathrm{d} \boldsymbol{x}}{\mathrm{d} t}=\boldsymbol{v}$.

Here, $\gamma=1 / \sqrt{1-v^{2} / c^{2}}$ is the relativistic Lorentz factor; $m$ and $q$ are the rest mass and the charge of the particle; $\boldsymbol{x}$ and $\boldsymbol{v}$ are the position and velocity vectors of the particle; $\boldsymbol{E}$ and $\boldsymbol{B}$ are the electric and magnetic fields that the particle experiences on its way. Taking the scalar product of $\boldsymbol{v}$ with the equation of motion (9), one gets the following equation for the change in kinetic energy

$\frac{\mathrm{d} E_{k}}{\mathrm{~d} t}=q \boldsymbol{v} \cdot \boldsymbol{E}$,

where $E_{k}=m c^{2}(\gamma-1)$ is the kinetic energy of the charged particle. According to Eq. (11), a charged particle can gain or lose kinetic energy in an electromagnetic field only if it has a velocity component in the direction of the electric field. In uniform magnetic fields, however, convective electric fields, which are always perpendicular to magnetic fields, cannot lead to the netgain of energy. This happens because the magnetized particle keeps gyrating and is accelerated and again decelerated within one gyro-period.

The possible way to accelerate particles in convective electric fields is therefore either to break down their magnetization, or to let them drift in the direction of the electric field due to the gradient and curvature force. De-magnetized particles can be directly accelerated by the electric field. Magnetized particles can only gain energy while undergoing a strong drift motion in the direction of the electric field.

In a non-uniform magnetic field, the magnetic-field gradient force $\boldsymbol{F}_{\text {grad }}=\left(m v_{\perp}^{2} / 2 B\right) \nabla \boldsymbol{B}$ and the curvature force $\boldsymbol{F}_{\text {curv }}=$ $\left(m v_{\|}^{2} / R^{2}\right) \boldsymbol{R}$ cause a drift motion perpendicular to both the magnetic field and the force direction. If there is a component of the drift velocity in the direction of the electric field, the particles could either gain or lose energy in the electric field according to Eq. (11). The gradient drift and the curvature drift velocities are

$\boldsymbol{v}_{\text {grad }}=\frac{m v_{\perp}^{2}}{2 q B^{3}} \boldsymbol{B} \times \nabla B$

$\boldsymbol{v}_{\text {curv }}=\frac{m v_{\|}^{2}}{q B^{2} R_{\text {curv }}^{2}} \boldsymbol{R}_{\text {curv }} \times \boldsymbol{B}=\frac{m v_{\|}^{2}}{q B^{2}} \boldsymbol{B} \times\left(\nabla \frac{\boldsymbol{B}}{|B|} \cdot \frac{\boldsymbol{B}}{|B|}\right)$.

These drift velocities vary inversely proportional to the magnitude of the magnetic field. Therefore particle drifts are maximum where the magnetic field is minimum and the gradients and curvatures are strong. A null point provides a highly probable location for these effects. For $m v_{\perp}^{2} / 2=m v_{\|}^{2} / 2=E_{k} / 2$ and $E_{k}=200 \mathrm{eV} \sim 2.32 \mathrm{MK}$ we calculated the drift velocities $\boldsymbol{v}_{\mathrm{grad}}$ and $\boldsymbol{v}_{\text {curv }}$ for the whole simulation box. We found that their maximum values are attained at the location 46.4, 46.4, 14.2 close to the null, which is $36.9 \mathrm{~m} / \mathrm{s}$ for $\boldsymbol{v}_{\text {grad }}$ and $21.6 \mathrm{~m} / \mathrm{s}$ for $\boldsymbol{v}_{\text {curv }}$. The resulting gradient and curvature drift velocities around the null obtained by solving Eqs. (12) and (13) are shown in Fig. 5. These drift velocities are estimated for particles with a thermal energy about $200 \mathrm{eV}$, which corresponds to thermal velocities of $1.95 \times 10^{5} \mathrm{~m} / \mathrm{s}$ for protons and $8.37 \times 10^{6} \mathrm{~m} / \mathrm{s}$ for electrons. Note that the drift velocities are small compared to the particle velocities, as required for the validity of Eqs. (12) and (13). Thereafter, once particles have been accelerated, their drift velocities will also be heightened, a feedback which favors further acceleration as in a snowball effect.

Moreover, the electric drift $\boldsymbol{v}_{E}=\boldsymbol{E} \times \boldsymbol{B} / B^{2}$ may also cause particle acceleration (e.g, Vekstein \& Browning 1997; Northrop 1963). When the particles move away from the null point, the continuous change of the magnetic and electric field will lead to the non-uniformity of the electric drift speed. As a result, particles will not follow the drift stream lines, and the acceleration is associated with the changing electric drift velocity.

In the course of this adiabatic drift-acceleration it may happen that the particle gyration radii $\left(L_{i}=m v /|q| B\right)$ become comparable to the scale lengths of the magnetic non-uniformity. In 

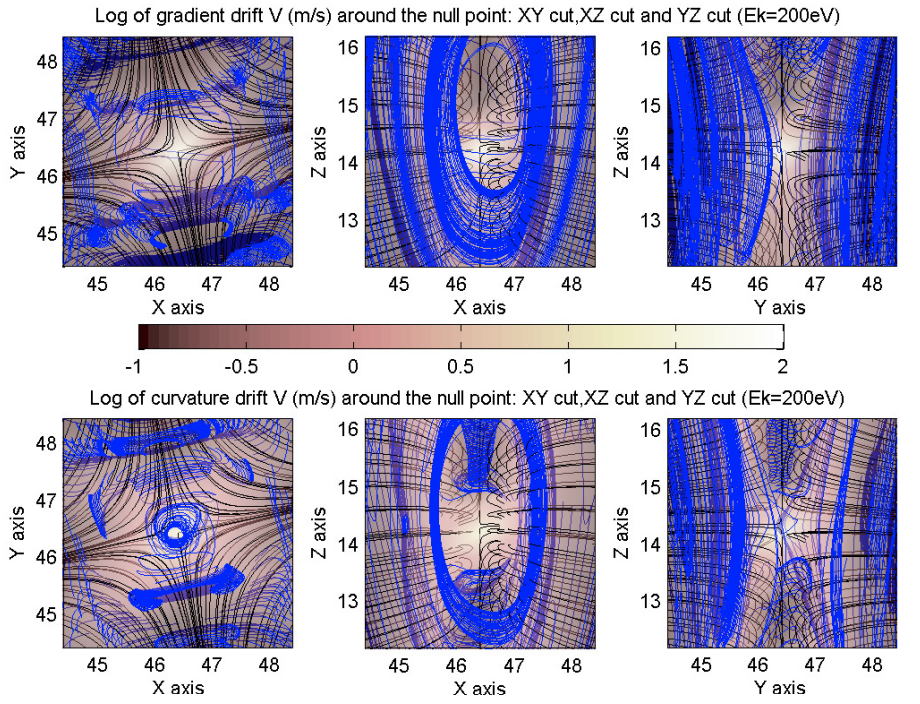

Fig. 5. Gradient (top) and curvature (bottom) drift lines (blue lines) around the null (magnetic field lines are shown as black lines). The left/middle/right column shows the projection of the flow lines in the $x-y / x-z / y-z 2$-D plane cutting through the null. The magnitude of the drift velocity in logarithmic-scale is shown in color with a brighter color indicating a higher drift speed.
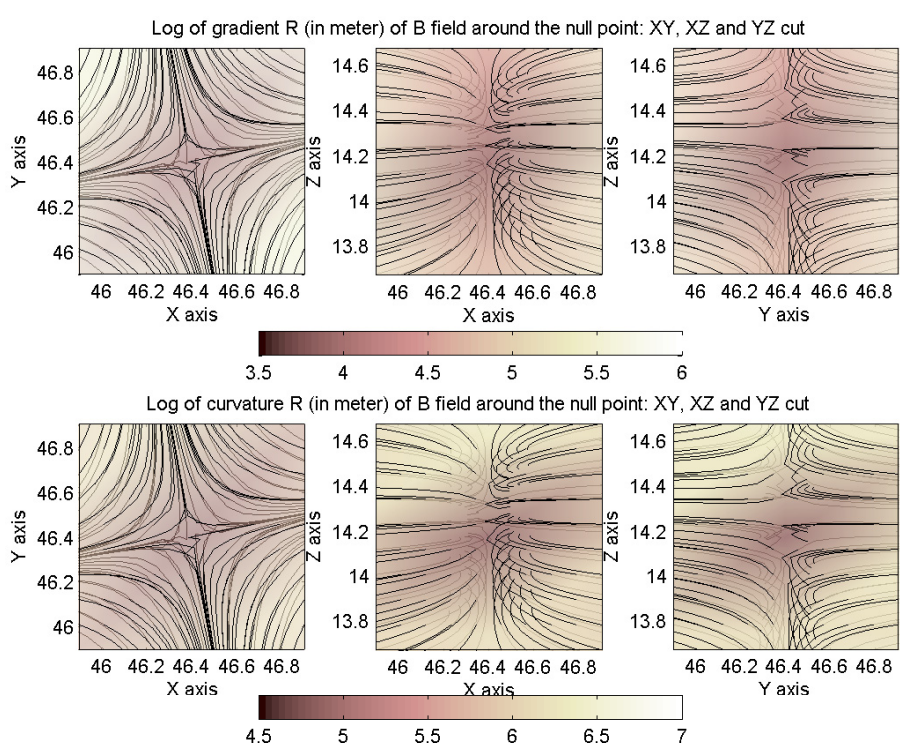

Fig. 6. Lengths of magnetic gradient scale (top) and curvature scale (bottom) around the null (magnetic fields are shown in black lines). The left/middle/right plot shows the $x-y / x-z / y-z$ plane cutting through the null. The magnitude of the scale lengths in the logarithmic-scale is color-coded with the darker color indicating a lower value.

this case particles lose their magnetization and adiabatic state and can be directly accelerated in the perpendicular electric field (e.g. Büchner 1986; Büchner \& Zelenyi 1989). A null point should be a prime candidate location for both drift and nonadiabatic acceleration. To verify this hypothesis quantitatively, we calculated the magnetic gradient and curvature scale according to $R_{\text {grad }}=|\boldsymbol{B}| /|\nabla \boldsymbol{B}|$ and $R_{\text {curv }}=|\boldsymbol{B}|^{3} /|\boldsymbol{B} \times(\nabla \boldsymbol{B} \cdot \boldsymbol{B})|$. Figure 6 displays the gradient and curvature scale lengths around the null. The average values of $R_{\text {grad }}$ and $R_{\text {curv }}$ around the null (where $B<1$ ) are $0.79 L_{0}$ and $5.18 L_{0}$ respectively. The minimum $R_{\text {grad }}$ and $R_{\text {curv }}$ in the whole MHD-simulation box are located near the

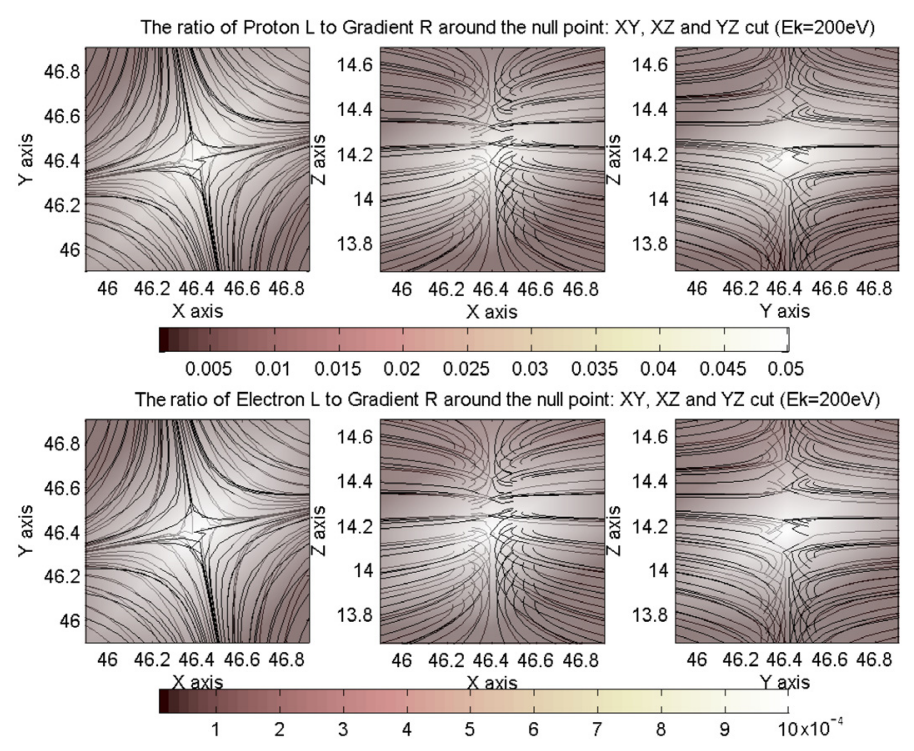

Fig. 7. Ratios of the Larmor radii to the the magnetic gradient scale lengths for a $200 \mathrm{eV}$ proton (top) and a $200 \mathrm{eV}$ electron (bottom) near the null (magnetic fields are shown in black lines). The left/middle/right column shows $x-y / x-z / y-z 2$-D cuts through the null. The magnitude of the ratios is shown in gray scale with a brighter color indicating a higher value.

null point, which is $0.0176 L_{0}$ and $0.1516 L_{0}$ i.e. $8.8 \mathrm{~km}$ and $76 \mathrm{~km}$. Notice that the calculation of the gradient and curvature scales is limited by the coarse grid sizes of the MHD box. In coronal fields, one should expect smaller non-uniform scales. On the other hand, the Larmor radius of a gyrating particle $(\sim v / B)$ is enlarged near the null by the weak magnetic field. Assuming a typical coronal particle energy of $200 \mathrm{eV}$, one obtains $L_{\mathrm{p}}$ about $20 \mathrm{~m}$ and $L_{\mathrm{e}}$ about $0.5 \mathrm{~m}$ for $B=1 \mathrm{G}$. At the location where $B=0.0484 \mathrm{G}$, the Larmor radii of protons and electrons are $L_{\mathrm{p}}=420 \mathrm{~m}$ and $L_{\mathrm{e}}=10 \mathrm{~m}$ respectively. The resulting ratios of the Larmor radii to the magnetic gradient scale lengths of both a $200 \mathrm{eV}$ proton (top) and a $200 \mathrm{eV}$ electron (bottom) are shown in Fig. 7. As one can see in the figure, near the null point Larmor radii and magnetic gradient scales are comparable for protons, while for electrons this ratio is much smaller. Therefore we can expect that protons will undergo stronger drifts and are more likely to be non-adiabatically accelerated than electrons. Note that once particle energies are increased, the Larmor radii will also be enlarged and the chance of de-magnetization and consequent direct acceleration will be significantly enhanced. To investigate how particles become accelerated near the null, we used a test-particle approach which we describe in next section.

\section{Simulation of particle acceleration near a 3-D null}

We assumed that the accelerated particles exert negligible feedback forces on the background coronal electromagnetic fields. Hence we could use a full-orbit relativistic test particle approach, in which the protons or electrons individually explore in the prescribed electromagnetic field as described and calculated in Sect. 2. We took a fixed snapshot of the macroscopic fields and freezed them during the evaluation of the particle trajectories.

We calculated the particle motion by numerically solving the relativistic momentum Eq. (9) together with (10). In order to find out whether in ideal MHD the convective electric field as theoretically described in Sect. 3 around the null is able to accelerate 
Table 1. Calculation of particle kinetic energies (in keV) under different electric fields.

\begin{tabular}{lllll}
\hline \hline Case & Acc. time & $\operatorname{Min} E_{k}$ & Aver $E_{k}$ & $\operatorname{Max} E_{k}$ \\
\hline 1: protons & $0.27 \mathrm{~s}$ & 0.2 & 0.22 & 0.25 \\
1: electrons & $0.007 \mathrm{~s}$ & 0.2 & 0.2005 & 0.201 \\
2: protons & $0.23 \mathrm{~s}$ & 0.17 & 0.53 & 0.95 \\
2: electrons & $0.007 \mathrm{~s}$ & 0.2 & 0.206 & 0.211 \\
3: protons & $0.1 \mathrm{~s}$ & 7.43 & 19.21 & 30.02 \\
3: electrons & $0.006 \mathrm{~s}$ & 0.2 & 0.26 & 0.32 \\
4: protons & $0.028 \mathrm{~s}$ & 1779.9 & 2083.6 & 2272.0 \\
4: electrons & $0.004 \mathrm{~s}$ & 0.16 & 1.34 & 2.73 \\
\hline
\end{tabular}

particles, we considered only the perpendicular convective electric field and neglected any parallel electric field. Of course, to obtain the field values at the particle position, one has to interpolate between the much more distant grid points of the MHD simulation. We used the eight neighbouring grid points nearest to a particle's position and applied a 3-D linear interpolation scheme to get the magnetic field and plasma velocities, which allowed us to determine the local convective electric field at the actual particle position. With a Runge-Kutta-Fehlberg fourth-fifth-order method with an adaptive time step, we integrated the positions and velocities of both protons and electrons in time.

We focused on the mechanism and process of particle acceleration by the null point, i.e. the highest energy a proton or electron could most probably gain from the convective electric field. The final acceleration spectrum, which depends on the initial spatial and velocity distributions, is not taken into account. Hence the initial launch point of particles was taken to be the point with the minimum magnetic field, where the particles are most likely to be accelerated. The initial velocities were shelldistributed: all the particles shared the same speed, but started in random directions. For the initial speed we first took the speed for particles with a kinetic energy of $200 \mathrm{eV}$ (corresponding to the most probable speed at a temperature of $2.32 \mathrm{MK}$ ), which yields $8.37 \times 10^{6} \mathrm{~m} / \mathrm{s}$ for electrons and $1.95 \times 10^{5} \mathrm{~m} / \mathrm{s}$ for protons. In Sect. 4.6, we also investigate the influence of higher initial energies on the acceleration process.

\subsection{Particle acceleration under different plasma flow conditions}

Since there is no direct observation of the magnitude of coronal convection and corresponding electric fields, we derived these quantities by the MHD simulation. The MHD equations were solved for dimensionless quantities so that we could rescale the dimensional values of the convection plasma flow $\boldsymbol{u}_{\text {con }}=u_{0} \boldsymbol{u}^{\prime}$ and the electric field $\boldsymbol{E}_{\text {con }}=E_{0} \boldsymbol{E}^{\prime}=u_{0} B_{0} \boldsymbol{E}^{\prime}$ by changing the normalization value $u_{0}$. The rescaling was not applied to $B_{0}$ so that the magnetic field strength was unaltered in the vicinity of the magnetic null point. Rescaling the plasma flow velocity corresponds to a change of the magnitude of the driving motions in the photosphere (at the bottom of the simulation box).

Table 1 shows the energies acquired by a certain number of particles (1000 particles in each case) with the initial energy $V_{\text {initial }}=200 \mathrm{eV}$ and the location $X_{\text {initial }}=(46.4,46.4,14.2)$ for different magnitudes of the plasma flow velocities and their corresponding different coronal convective electric fields. "Acc.time" represents the acceleration time which was defined so that the minimum energy (Min $E_{k}$ ), average energy (Aver $E_{k}$ ) and maximum energy (Max $E_{k}$ ) of a group of particles (1000 particles in each case) did not show any obvious changes after this initial acceleration phase. The average plasma flow velocities imposed at the bottom, $\bar{u}_{z=0}$, increased from Case 1 to Case 4 , being $0.69,6.9,69$, and $690 \mathrm{~km} \mathrm{~s}^{-1}$ respectively. The plasma flow velocities at the reference null, $u_{\text {null }}$, are 11.64, $116.4,1164$, and $11640 \mathrm{~km} \mathrm{~s}^{-1}$ from Case 1 to 4 . The corresponding convection electric fields $E_{\text {null }}$ are enhanced from Case 1 to 4 : being $0.0181,0.181,1.81$, and $18.1 \mathrm{~V} / \mathrm{m}$ respectively. $\bar{u}_{B<2}$ (being 7.52, 75.2, 752, $7520 \mathrm{~km} \mathrm{~s}^{-1}$ from Case 1 to 4 ) and $\bar{E}_{B<2}$ (being $1.15,11.5,115,1150 \mathrm{~V} / \mathrm{m}$ from Case 1 to 4 ) are the average plasma velocities and convective electric fields around the null area $(B<2 \mathrm{G})$.

One thousand protons were launched near the null for each case of plasma conditions. In Case 1, the plasma flow (convection) velocity at the reference null point was $11.64 \mathrm{~km} \mathrm{~s}^{-1}$ and the average plasma velocity inside the area where $B<2 \mathrm{G}$ was $7.52 \mathrm{~km} \mathrm{~s}^{-1}$. The corresponding convective electric fields were $0.0181 \mathrm{~V} / \mathrm{m}$ at the point and $1.15 \mathrm{~V} / \mathrm{m}$ on average. After $0.27 \mathrm{~s}$ of motion in the electromagnetic fields, the protons' energies were only slightly enhanced. In Case 2, with stronger driving at the bottom, both the plasma velocity and electric field were ten times higher and more extended than in Case 1. Protons were moderately accelerated within $0.23 \mathrm{~s}$, and the maximum energy nearly reached a value of $1 \mathrm{keV}$. In Case 3, the bottom driving plasma velocity was on average $70 \mathrm{~km} \mathrm{~s}^{-1}$ and the plasma velocity at the reference null point was $1164 \mathrm{~km} \mathrm{~s}^{-1}$, a number that is reasonable for the magnetically active coronal environment. Correspondingly, the convective electric field was $1.81 \mathrm{~V} / \mathrm{m}$ at the grid. It is shown that protons are efficiently accelerated up to $30 \mathrm{keV}$ within $0.1 \mathrm{~s}$. Case 4 corresponds to the situation of very strong driving (driving velocity being $700 \mathrm{~km} \mathrm{~s}^{-1}$ on average), which can be assumed for a powerful flare explosion. The resulting convective electric field was $18.1 \mathrm{~V} / \mathrm{m}$ at the reference null point and $1150 \mathrm{~V} / \mathrm{m}$ on average inside the area $(B<2 \mathrm{G})$. This situation allowed a proton to be accelerated to an energy of more than $2 \mathrm{MeV}$.

For electrons, however, no acceleration was achieved in Case 1 and Case 2. In Case 3, i.e. in active regions, their kinetic energy was slightly enhanced to $300 \mathrm{eV}$. Case 4 shows that electrons near coronal nulls of explosive flare conditions may be accelerated to energies close to $3 \mathrm{keV}$. Electron acceleration by a perpendicular convective electric field is less efficient, because their Larmor radii are much smaller than the nonuniformity scale of the magnetic field (bottom images in Fig. 7), and therefore it is very difficult to de-magnetize electrons. Their efficient acceleration may require a parallel electric field, which can accelerate particles adiabatically along the magnetic field lines (Litvinenko 1996). However, the inclusion of parallel electric fields necessitates to consider kinetic processes that enable their formation and determine their spatial scale.

\subsection{Single proton orbit study for Case 3}

In order to understand acceleration near the null, we considered the evolution of orbits, kinetic energies, pitch angles and Larmor radii for a single proton in Case 3 (active region).

Figure 8 shows a typical proton orbit during the first $0.1 \mathrm{~s}$ of acceleration. The spatial distance between two neighboring asterisks, marking $0.02 \mathrm{~s}$ time intervals, is increasing. This indicates that the proton is moving faster and faster. The gray scale shows the magnitude of the magnetic field, which increases away from the null. Figure 9 shows the evolution of the particle's kinetic energy, the velocity-magnetic field $(V-B)$ pitch angle, the velocity-electric field $(V-E)$ angle, the strength of magnetic and electric fields, and the logarithmic value of curvature scale, 


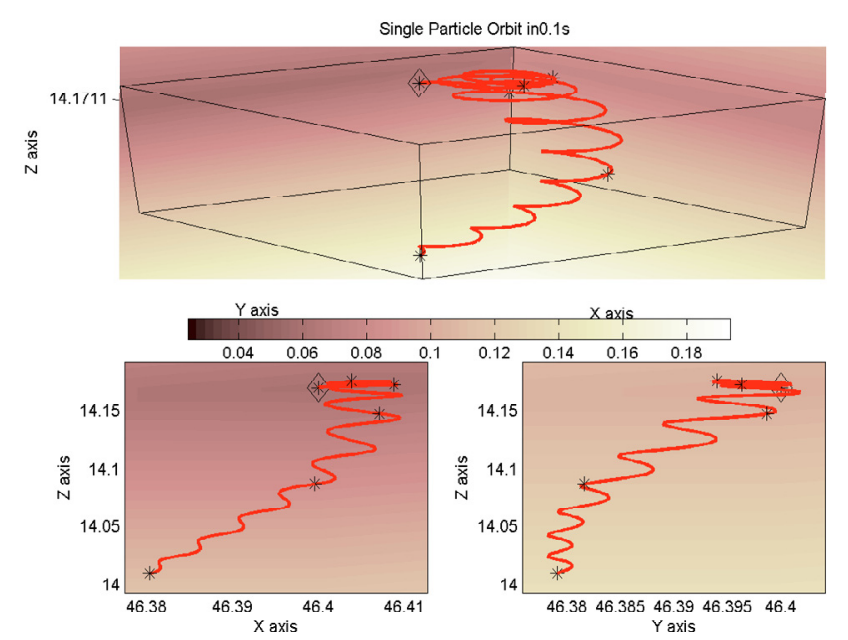

Fig. 8. Orbit of a single proton during $0.1 \mathrm{~s}$ of acceleration (Case 3, active region). Top image: $3-\mathrm{D}$ view of the orbit. Bottom images: $x-z$ (left) and $y-z$ (right) projection views. The square-asterisk dotted shows the starting point of the particle, while other asterisks show the locations after each time step of $0.02 \mathrm{~s}$. The gray scale represents the strength of the magnetic field, which increases while the particle moves out of the null.
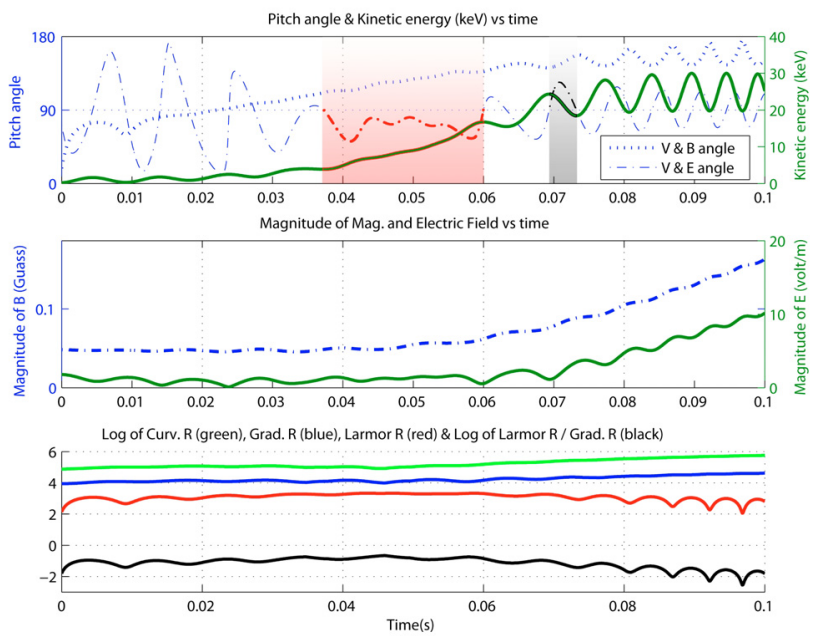

Fig. 9. Acceleration of a single proton during $0.1 \mathrm{~s}$ (Case 3, active region). Top panel: the green solid line shows the energy-gain; the blue dotted and dashdotted lines represent the angle of the proton velocity with respect to the magnetic and electric field. The red/gray high lighted regions give examples of the acceleration/deceleration process, whereby the energy increases/decreases and the angle between velocity and electric field is narrower/wider than 90 degrees. The middle image shows the local magnitude of the magnetic field $(\mathrm{G})$ and electric field $(\mathrm{V} / \mathrm{m})$ on the way of the proton orbit. The bottom image shows the logarithmic value of the curvature scale, gradient scale, Larmor radius and the ratio of the Larmor radius to the gradient scale. All length values are in meters.

gradient scale, Larmor radius and the ratio of Larmor radius to gradient scale. The red high lighted region in the top image indicates the interval of major acceleration, during which the kinetic energy is increasing and the $V-E$ angle for several gyro-periods remains smaller than 90 degrees. This means that the proton is de-magnetized and moves non-adiabatically in the direction of the electric field. The proton is therefore efficiently accelerated during this process. The gray high-lighted region, on the other hand, corresponds to an example of a deceleration moment due to the gyro-motion. The $V-E$ angle stays wider than 90 degrees,
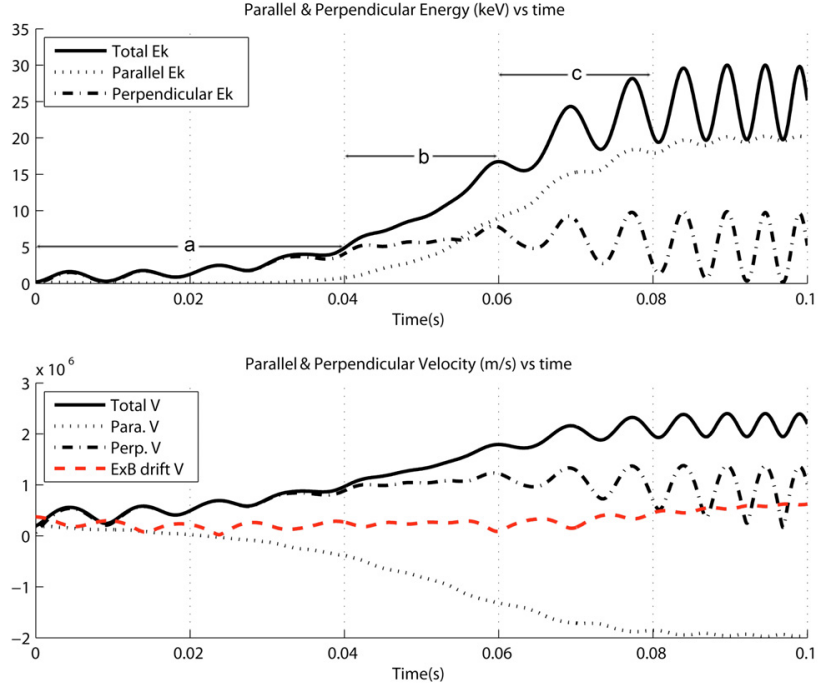

Fig. 10. Kinetic energy (top) and velocity (bottom) evolutions in both parallel (dotted lines) and perpendicular (dash-dotted lines) directions of a single proton during $0.1 \mathrm{~s}$ are shown under Case 3 conditions. Solid lines are showing the total kinetic energy (top) and velocity (bottom). Also the electric drift velocity is shown in dashed line (bottom).

meaning that the proton moves against the direction of the electric field.

During the initial $0.07 \mathrm{~s}$, the average Larmor radius (red line in bottom image of Fig. 9) was about $2 \mathrm{~km}$, and the proton underwent strong drifts with a transition to non-adiabatic motion (from $0.04 \mathrm{~s}$ to $0.06 \mathrm{~s}$ ) after initial acceleration. As the proton was moving away from the null, the magnetic field increased (middle image in Fig. 9) and the particle started to be magnetized with a regular gyration and smaller gyro-radii. The bottom panel of Fig. 9 shows that both curvature and gradient scale lengths were increasing, while the Larmor radius decreased because the proton moved towards the stronger magnetic field. When the ratio of Larmor radius to gradient scale dropped below $10^{-1}$ and the main acceleration phase was finished, the proton became remagnetized. Notice that magnetic fields from a MHD simulation would significantly smooth small scale non-uniform structures, a process which is very important for demagnetization. This nonadiabatic acceleration process would be more effective if microscopic turbulence of the magnetic field were considered.

Figure 10 separately shows the parallel and perpendicular components of both the kinetic energy and velocity of the particle. There are three different phases of acceleration: (a) perpendicular acceleration due to (mostly gradient) drift within the first $0.04 \mathrm{~s}$, (b) parallel acceleration due to non-adiabatic motion from $0.04 \mathrm{~s}$ to $0.06 \mathrm{~s}$, and (c) parallel acceleration due to (mainly curvature) drift from $0.06 \mathrm{~s}$ to $0.08 \mathrm{~s}$. We show in Fig. 10 that the parallel kinetic energy stayed relatively small during the initial $0.04 \mathrm{~s}$, and only the perpendicular energy was enhanced from less than $0.2 \mathrm{keV}$ to about $5 \mathrm{keV}$. This is because the convection electric field is perpendicular to the magnetic field, and thus the perpendicular energy can easily be enhanced. The gradient drift velocity (Eq. (12)) was hence enlarged and helped the drift acceleration as a feedback. This initial acceleration prepared the particles for the subsequent non-adiabatic acceleration phase.

From $0.04 \mathrm{~s}$ to $0.06 \mathrm{~s}$, the proton did not complete full gyrations (de-magnetized) and therefore could be continuously accelerated in the electric field (also shown by the small $V-E$ angle in Fig. 9). The perpendicular energy almost stopped growing, while the parallel energy increased a lot (up to $9 \mathrm{keV}$ at 

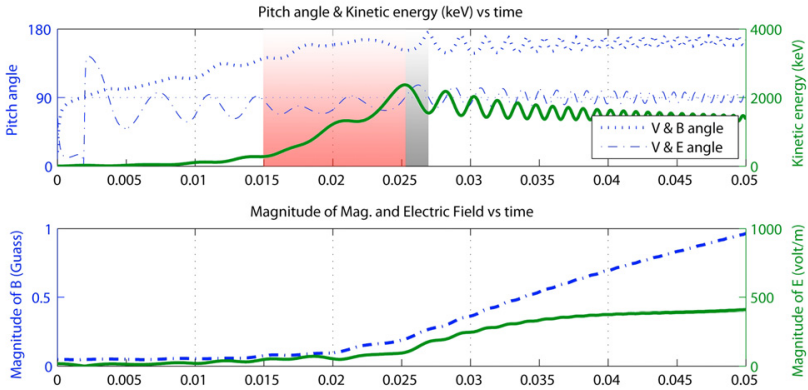

Log of Curv. R (green), Grad. R (blue), Larmor R (red) \& Log of Larmor R/ Grad. R (black)

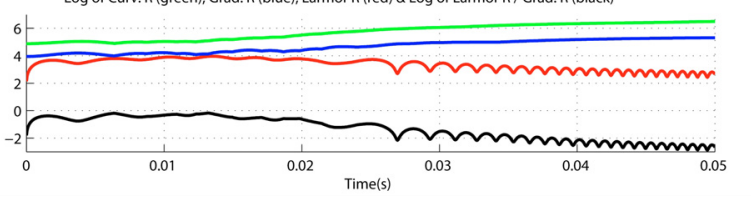

Fig. 11. Energy evolution of a single proton during $0.05 \mathrm{~s}$ under Case 4 conditions. In the top image, the green solid line shows the energygaining process. The blue dotted and dash-dotted lines show the $V-B$ angle and $V-E$ angle. The red/gray high-lighted regions give examples of the acceleration/deceleration process, whereby the energy is increasing/decreasing and the $V-E$ angle is narrower/wider than 90 degrees. The middle image shows the local magnitude of the magnetic field $(\mathrm{G})$ and electric field $(\mathrm{V} / \mathrm{m})$ on the way of the proton orbit. The bottom image shows the logarithmic value of the curvature scale, gradient scale, Larmor radius and the ratio of the Larmor radius to the gradient scale. All length values are in meters.

$0.06 \mathrm{~s})$. This non-adiabatic process transfered the perpendicular energy immediately into parallel energy. When the proton was re-magnetized at $0.06 \mathrm{~s}$, this high parallel energy enhanced the curvature drift (Eq. (13)), causing further acceleration in the parallel direction (from $9 \mathrm{keV}$ at $0.06 \mathrm{~s}$ to $18 \mathrm{keV}$ at $0.08 \mathrm{~s}$ ), until the proton stopped its perpendicular drift at $0.08 \mathrm{~s}$. The parallel energy stayed around $20 \mathrm{keV}$, and the perpendicular energy oscillated around $5 \mathrm{keV}$. Hence the total energy was about $25 \mathrm{keV}$.

After $0.08 \mathrm{~s}$, the proton pitch $(V-B)$ angle was approaching 180 degrees (see the top panel of Fig. 9). This indicates that the proton could escape along (albeit against the direction of) the magnetic field downward to the photosphere. The $V-E$ angle stayed around 90 degrees in the end. Hence the acceleration was finished. The final particle velocity was the sum of three types of motion: parallel velocity, perpendicular drift velocity and perpendicular gyration velocity. The last part was apparent as an oscillation (especially after $0.07 \mathrm{~s}$ when the proton is magnetized) of the perpendicular velocity and energy (Fig. 10) as well as of the $V-B$ and $V-E$ angle (top panel of Fig. 9). After averaging it over the gyration period, the remaining perpendicular velocity is the drift velocity, which is mostly due to the $\boldsymbol{E} \times \boldsymbol{B}$ drift $\left(E_{\perp} / B=E / B\right)$ in the end. As one can see in the bottom frame of Fig. 10, the electric drift velocity (dashed line) approached the average of the perpendicular velocity (dash-dotted line) after $0.08 \mathrm{~s}$ and about $600 \mathrm{~km} \mathrm{~s}^{-1}$ at $0.1 \mathrm{~s}$. This electric drift velocity is consistent with the perpendicular component of the plasma velocity as obtained from the MHD simulation i.e. $\boldsymbol{E}_{\mathrm{con}}=-\boldsymbol{u} \times \boldsymbol{B}$.

\subsection{Single proton orbit study for Case 4}

In order to understand how protons could be accelerated up to $2 \mathrm{MeV}$ during flares (Case 4 conditions - strong convection which could only happen during big flare explosions), we again study the acceleration process of a typical single proton.
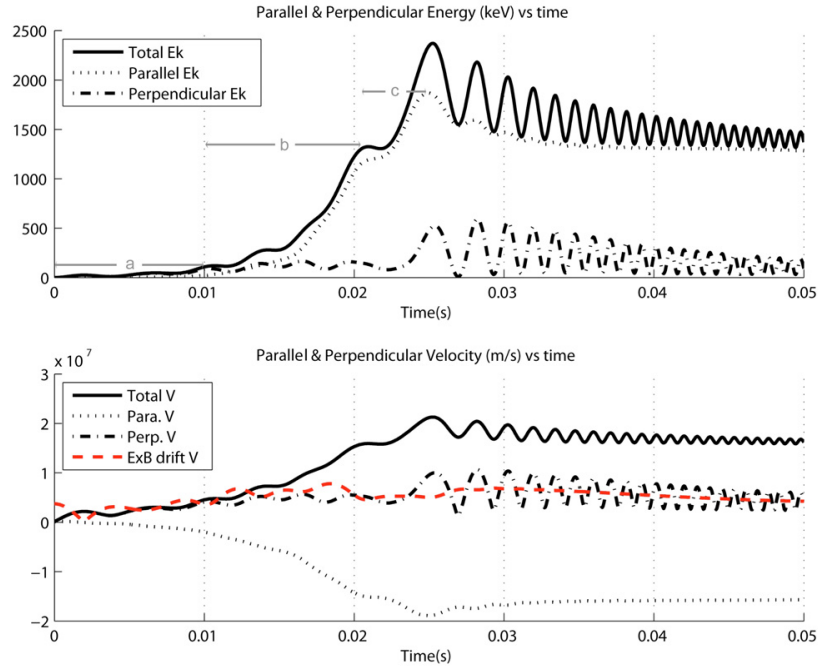

Fig. 12. Under Case 4 conditions, the evolutions during $0.05 \mathrm{~s}$ of the kinetic energy (top) and velocity (bottom) in both parallel (dotted lines) and perpendicular (dash-dotted lines) directions of a single proton are shown. Solid lines represent the total kinetic energy (top) and magnitude of velocity (bottom). Also the electric drift velocity is shown as a dashed line (bottom).

Figure 11 shows the evolutions of the $V-B$ and $V-E$ angle, local magnetic and electric field strength, gradient and curvature scale and Larmor radius. As in Case 3, the red high-lighted region in the top image indicates the main non-adiabatic acceleration phase, during which the proton is continuously accelerated. The gray high lighted region indicates an example of deceleration phase due to gyro-rotation. Figure 12 represents the evolutions of the parallel and perpendicular energies and velocities separately.

It is clear that the acceleration process is much more efficient than that of Case 3 , because the electric field is much stronger and the proton is less magnetized. The Larmor radius of the proton during the first $0.02 \mathrm{~s}$ (about $6 \mathrm{~km}$ ) is very close to the gradient scale (about $10 \mathrm{~km}$ ), as one can see at the bottom panel of Fig. 11. As in Case 3, Fig. 12 shows that there are also three different phases of acceleration in Case 4: (a) perpendicular acceleration within the first $0.01 \mathrm{~s}$, during which mainly the perpendicular energy is enhanced (from less than $200 \mathrm{eV}$ to nearly $100 \mathrm{keV}$ at $0.01 \mathrm{~s}$ ), (b) parallel acceleration due to non-adiabatic motion from $0.01 \mathrm{~s}$ to $0.021 \mathrm{~s}$, whereby the parallel energy increases dramatically (from about $20 \mathrm{keV}$ at $0.01 \mathrm{~s}$ to $1200 \mathrm{keV}$ at $0.021 \mathrm{~s})$, (c) parallel acceleration due to (mainly curvature) drift from $0.021 \mathrm{~s}$ to $0.025 \mathrm{~s}$, whereby the parallel energy keeps increasing albeit at a lower speed (to about $1850 \mathrm{keV}$ at $0.025 \mathrm{~s}$ ) when the proton is re-magnetized. The parallel energy is then slightly decreased after $0.025 \mathrm{~s}$ though, because the magnetic field increases while the proton moves out from the null (see middle panel of Fig. 11), and the parallel energy is transferred to perpendicular energy by the magnetic-mirror effect. Finally (at $0.05 \mathrm{~s}$ ), the parallel energy reaches $1.3 \mathrm{MeV}$ (see the dotted line in the top panel of Fig. 12). The perpendicular energy, which includes mainly the electric drift (see the red dashed line in the bottom panel of Fig. 12) is oscillating (due to the gyration) around $100 \mathrm{keV}$. The final high parallel velocity (i.e. small pitch angle: $V-B$ is close to 180 degrees) lets the proton escape from the null along the field lines. 

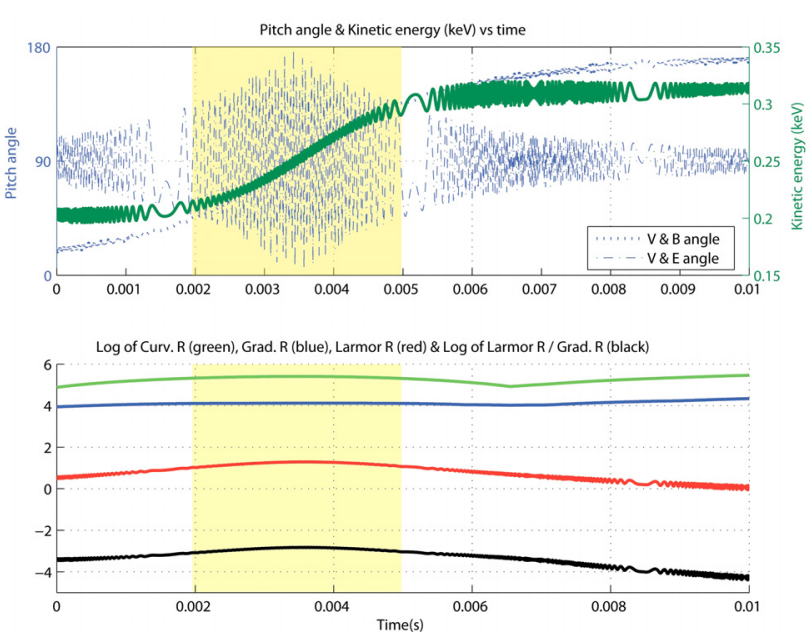

Fig. 13. Top panel shows the evolution of the kinetic energy and the pitch angles for a typical electron accelerated in active corona (Case 3 condition). The green thick line shows the kinetic energy and the blue dotted and dash-dotted lines represent the $V-B$ and $V-E$ angles respectively. The bottom panel shows the logarithmic value of curvature and gradient scales, electron Larmor radii and the ratio of Larmor radii to gradient scales. All lengths are given in meters.
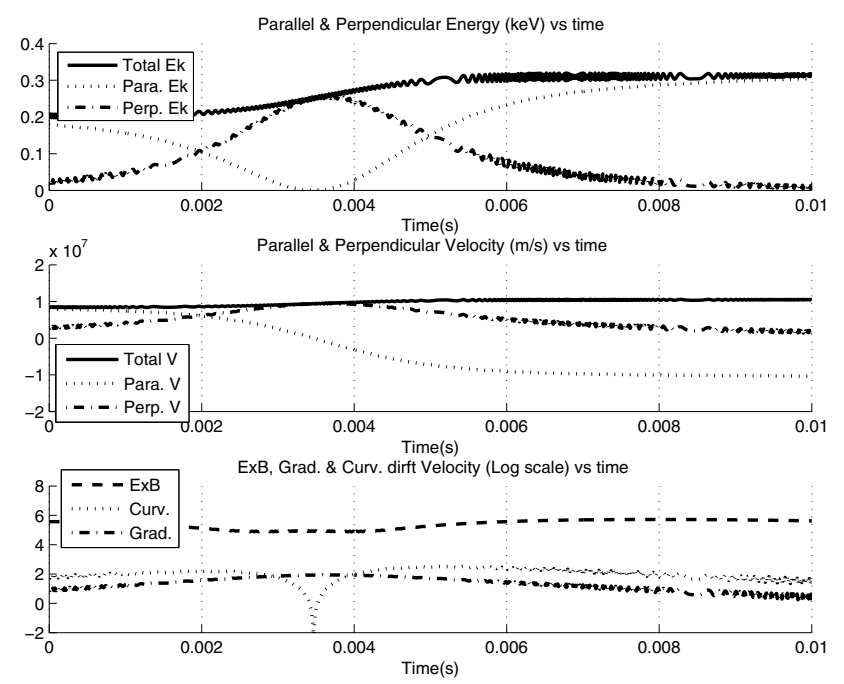

Fig. 14. In active regions (Case 3), the kinetic energy (top panel) and velocity (middle panel) evolutions in both parallel (dotted lines) and perpendicular (dash-dotted lines) directions of a single electron are shown. Solid lines represent the total kinetic energy (top panel) and velocity (middle panel). In the bottom panel, the electric drift velocity is shown as a dashed line and the gradient and curvature drift velocities are indicated as dotted and dash-dotted lines.

\subsection{Single electron orbit study for Case 3}

Similar to the single proton acceleration, we also investigated a single electron orbit (Figs. 13 and 14) for Case 3 (active region) conditions. We found that the electrons were not significantly accelerated. Their kinetic energies were only slightly enhanced due to drifts. Within about $0.006 \mathrm{~s}$, the electron kinetic energy increased from $200 \mathrm{eV}$ to just a little more than $300 \mathrm{eV}$. During the main acceleration (yellow highlighted region in the figure), the ratio of the Larmor radius to the gradient scale was about $10^{-3}$ (bottom panel of Fig. 13), and the $V-E$ pitch angle indicated a strong parallel (particle velocity parallel to the electric field) component (top panel of Fig. 13).

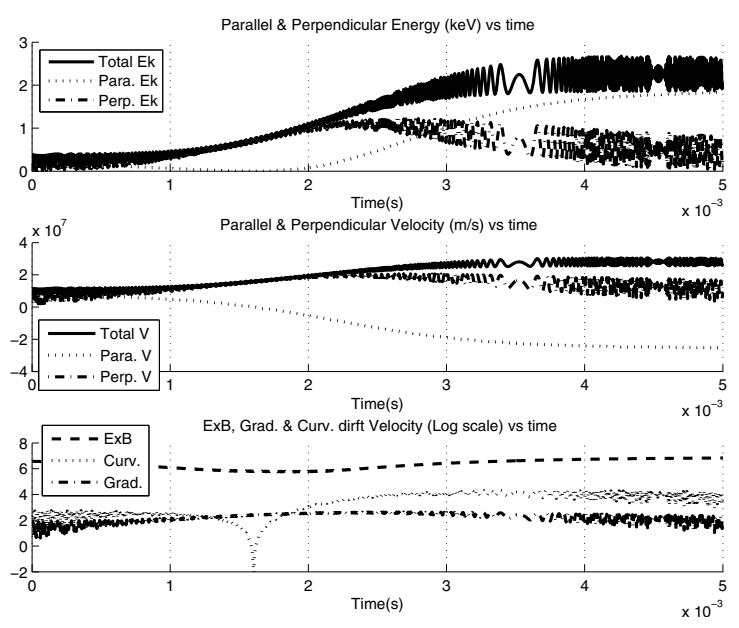

Fig. 15. In solar flares (Case 4), the kinetic energy (top panel) and velocity (middle panel) evolutions in both parallel (dotted lines) and perpendicular (dash-dotted lines) directions of a typical electron are shown. Solid lines represent the total kinetic energy (top panel) and velocity (middle panel). In the bottom panel, the electric drift velocity is shown as a dashed line and the gradient and curvature drift velocities are indicated as dotted and dash-dotted lines.

However, the electron did not have a non-adiabatic phase of acceleration due to its much smaller Larmor radius $(\sim 10 \mathrm{~m})$ and much shorter gyro-period $\left(\sim 10^{-7} \mathrm{~s}\right)$. Another reason for the absence of de-magnetization process could be that MHD simulation drops the information of small scale non-uniformity of magnetic fields. The electrons considered here do not have an awareness of the obvious change of the magnetic structure within several gyration periods. In the real solar corona we expect them to have a higher probability to experience nonadiabatic processes and thus to become accelerated.

Note that the electron shown in Fig. 14 initially carries mainly parallel energy. During the first $0.0035 \mathrm{~s}$, the perpendicular energy was increasing while the parallel energy decreased. At about $0.0035 \mathrm{~s}$, the parallel velocity changed its sign from parallel to anti-parallel and the curvature drift velocity reached zero according to Eq. (13) when $v_{\|}=0$. The bottom panel in Fig. 14 displays the velocities in logarithmic scale so that the zero value of the curvature drift velocity cannot be shown. Till $0.008 \mathrm{~s}$, the parallel energy grew back while the perpendicular energy decreased. This is similar to a magnetic-mirror effect, which means that when a particle moves into a stronger magnetic field, its parallel energy is transferred to its perpendicular energy until the parallel velocity reaches zero. Then the parallel velocity changes its sign, indicating that the electron is reflected and comes back towards the weaker magnetic field, so that the perpendicular energy starts being transferred to parallel energy. However, the magnetic moment $\left(E k_{\perp} / B\right)$ is not conserved during this process, because our 3 -D numerical magnetic field is very complex and the electric field is included. A particle experiences not only gradient and curvature drift, but also electric drift. These drift motions change the gyro-center orbit along which the electric field could change the kinetic energy as well as the magnetic moment.

\subsection{Single electron orbit study for Case 4}

Finally, electron acceleration in flares (Case 4) is shown in Fig. 15. There are mainly two phases of acceleration: perpendicular acceleration due to drift until $2 \times 10^{-3} \mathrm{~s}$ and parallel 
Table 2. Gradient and curvature drift velocities (in $\mathrm{m} / \mathrm{s}$ ), gradient and curvature scales (in $\mathrm{m}$ ) and Larmor radii (in $\mathrm{m}$ ) of both protons and electrons at the reference null.

\begin{tabular}{llllll}
\hline \hline At the null & $V_{\text {grad }}$ & $V_{\text {curv }}$ & $R_{\text {grad }}$ & $R_{\text {curv }}$ & $R_{\text {Larmor }}$ \\
\hline $200 \mathrm{eV}$ proton & 36.9 & 21.6 & 8778 & 75807 & 422.34 \\
$200 \mathrm{eV}$ electron & - & - & - & - & 9.85 \\
$2 \mathrm{keV}$ proton & 369 & 216 & - & - & 1335.6 \\
$2 \mathrm{keV}$ electron & - & - & - & - & 31.2 \\
$10 \mathrm{keV}$ proton & 1850 & 1080 & - & - & 2986.4 \\
$10 \mathrm{keV}$ electron & - & - & - & - & 69.7 \\
\hline
\end{tabular}

acceleration also due to drift till to $4 \times 10^{-3} \mathrm{~s}$. Because of the small electron Larmor radii and the absence of microscopic magnetic structures, there is no phase of non-adiabatic acceleration during which the electron is de-magnetized and continuously accelerated. Nevertheless, a net gain of energy due to the drift motion enhanced the total electron energy from $0.2 \mathrm{keV}$ to more than $2 \mathrm{keV}$. The final high parallel velocity causes the electron to escape from the null.

\subsection{Influence of the initial energy}

We also investigated the influence of the initial particle energy on the acceleration process. Higher initial energies with larger $v_{\perp}$ and $v_{\|}$reveal higher drift velocities (see Eqs. (12) and (13)) as well as larger Larmor radii. Hence particles can become more easily de-magnetized (Table 2). With the assumption that particles have already been pre-accelerated, we checked the possibility of a secondary acceleration by the null under the plasma flow conditions given in Case 3 (typical for active regions: $\bar{U}_{z=0}=69 \mathrm{~km} \mathrm{~s}^{-1}, U_{\text {null }}=1164 \mathrm{~km} \mathrm{~s}^{-1}, \bar{U}_{B<2}=752 \mathrm{~km} \mathrm{~s}^{-1}$, $E_{\text {null }}=1.81 \mathrm{~V} / \mathrm{m}$ and $\bar{E}_{B<2}=115 \mathrm{~V} / \mathrm{m}$.).

Table 3 summarizes the changes in kinetic energy for both protons and electrons and for different initial speeds. As one can see, for higher initial energies the final maximum kinetic energy is enhanced due to higher drift velocities and the acceleration time is shorter. However, we notice that protons can even be decelerated (shown as Min $E_{k}$ ) for higher initial energies. This is because these protons have an initial velocity direction opposite to the electric field, and the high initial energy pushes them far along this direction so that they may lose kinetic energy according to Eq. (11). With higher initial energies, most protons have a chance to be accelerated more strongly, while a few become decelerated.

For electrons, on the other hand, an enhanced initial kinetic energy does neither de-magnetize them nor switch on more efficient non-adiabatic acceleration, because the initial electron Larmor radii are only slightly enhanced from $\sim 10 \mathrm{~m}$ to $\sim 70 \mathrm{~m}$ (Table 2). These larger radii are still too small compared to the magnetic non-uniformity scale, which is obtained by macroscopic MHD simulations. Therefore secondary acceleration of electrons by convection electric fields cannot be expected at least in active regions (Case 3 ).

\section{Summary and discussion}

Using numerical MHD simulations, we investigated the 3-D field structure and plasma flows around a coronal magnetic null point (Sect. 2). Based on the interpolated structure of magnetic fields around the null, we theoretically described how particles can be accelerated by a convective electric field perpendicular to the magnetic field due to the magnetic gradient and curvature
Table 3. Calculations of particle kinetic energies (in keV) under different $V_{\text {initial }}\left(V_{i}\right)$ conditions.

\begin{tabular}{lllll}
\hline \hline & Acc. time & Min $E_{k}$ & Aver $E_{k}$ & $\operatorname{Max} E_{k}$ \\
\hline$V_{i}=200 \mathrm{eV}$ & & & & \\
$10^{3}$ protons & $0.1 \mathrm{~s}$ & 7.43 & 19.21 & 30.02 \\
$10^{3}$ electrons & $0.006 \mathrm{~s}$ & 0.198 & 0.258 & 0.323 \\
$V_{i}=2 \mathrm{keV}$ & & & & \\
$10^{3}$ protons & $0.056 \mathrm{~s}$ & 1.26 & 19.85 & 44.24 \\
$10^{3}$ electrons & $0.0024 \mathrm{~s}$ & 1.99 & 2.17 & 2.36 \\
$V_{i}=10 \mathrm{keV}$ & & & & \\
$10^{3}$ protons & $0.032 \mathrm{~s}$ & 8.49 & 27.66 & 63.37 \\
$10^{3}$ electrons & $0.001 \mathrm{~s}$ & 9.89 & 10.28 & 10.68 \\
\hline
\end{tabular}

drifts in Sect. 3. Finally, test particle calculations in Sect. 4 for both protons and electrons near the null provided a quantitative estimate of the particle acceleration process. Our main results can be summarized as follows.

- With extrapolated magnetic fields as the initial condition, our MHD simulation evolved to a state that plasma flow velocity and convective electric fields were generated through the whole simulation box. In the box center (with the height of solar corona region) the minimum magnetic field being less than $0.05 \mathrm{G}$ indicated the immediate vicinity of a magnetic null point. When rescaled to active region conditions (Case 3), the plasma flow velocity was about $1000 \mathrm{~km} \mathrm{~s}^{-1}$ and the corresponding convective electric field was about $2 \mathrm{~V} / \mathrm{m}$ at the null. Protons could be accelerated up to $30 \mathrm{keV}$ in $0.1 \mathrm{~s}$ and electrons gained only small energies, i.e. from $0.2 \mathrm{keV}$ to $0.3 \mathrm{keV}$.

- With higher plasma flow velocities and stronger convective electric fields ( $\sim 20 \mathrm{~V} / \mathrm{m}$ near the null), protons could be accelerated to energies up to $2 \mathrm{MeV}$ in $0.03 \mathrm{~s}$ and electrons were accelerated to about $3 \mathrm{keV}$ in $0.005 \mathrm{~s}$.

- The magnetic curvature radii $\left(\sim 10^{5} \mathrm{~m}\right)$ near the null exceeded the gradient scale lengths $\left(\sim 10^{4} \mathrm{~m}\right)$. Therefore gradient drifts favored the drifting acceleration more than the curvature drifts in the beginning of the acceleration. However, after the particles' parallel energy became much higher than the perpendicular energy, the curvature drift became more important than the gradient drift.

- During the major acceleration process the Larmor radii were about $10^{3} \mathrm{~m}$ to $10^{4} \mathrm{~m}$ for protons and $10 \mathrm{~m}$ to $100 \mathrm{~m}$ for electrons. The ratio of Larmor radii to gradient scale was about 0.1 to 1 for protons, but merely 0.001 to 0.01 for electrons. Non-adiabatic acceleration stopped when this ratio was smaller than 0.1 for protons. Nevertheless, since the non-uniformity scales obtained from MHD simulations are limited by the coarse grid sizes, one could expect the existence of even more non-uniform structures in realistic solar coronal fields, representing non-adiabatic regions and thus producing more effective acceleration.

- When the $V-E$ angle was less than 90 degrees over several gyro-periods, the proton could be accelerated continuously, which is known as non-adiabatic acceleration. When the particle was magnetized, deceleration could also occur because of the gyration around magnetic field lines.

- For protons, there were mainly three different phases of acceleration: (a) perpendicular acceleration due to (mostly gradient) drift, (b) parallel acceleration due to nonadiabatic motion and (c) parallel acceleration due to (mainly 
curvature) drift. The non-adiabatic phase yields the most efficient acceleration.

- For electrons, there were only two phases of acceleration: (a) perpendicular acceleration and (b) parallel acceleration, both due to drifts. Due to much smaller electron Larmor radii and also because of the absence of microscopic magnetic structures in macroscopic MHD simulations, the electrons were not aware of any obvious change of the magnetic field within several gyrations, and consequently there was no phase of non-adiabatic acceleration whereby they could be de-magnetized and continuously accelerated.

- The final particle velocity included three parts: parallel velocity, perpendicular drift velocity and perpendicular gyration velocity. The last term appeared as oscillations as particles were re-magnetized. The remaining perpendicular velocity was the drift velocity, which is partly due to gradient and curvature drifts during acceleration, but mainly stems from the $\boldsymbol{E} \times \boldsymbol{B} \operatorname{drift}\left(E_{\perp} / B=E / B\right)$ after re-magnetization of the protons. For electrons the $\boldsymbol{E} \times \boldsymbol{B}$ drift was always much larger than the gradient and curvature drifts. Hence electrons were not accelerated significantly near the null.

- For both protons and electrons, the final parallel velocities were much higher than the perpendicular velocities and the $V-B$ pitch angles were approaching 180 degrees. This means that the particles escaped from the null along the magnetic field lines outward and downward to the photosphere. Particles did not escape to the upper corona because of the non-symmetric configuration of the coronal magnetic field, which is weaker at higher altitudes and has fewer up-going open field lines connecting to the null.

- For higer initial kinetic energies (resulting in enhanced drifts), some protons had the chance to gain more energies in the course of a secondary acceleration process. Nevertheless, a few protons ran the risk to be decelerated (if their initial velocity was opposite to the direction of the electric field). For electrons, a higer initial energy did not lead to efficient acceleration, because they still remained strongly magnetized.

- Electrons could not become de-magnetized, owing to their smaller gyro-radii. Protons could not be accelerated to observational energy bands of about $10 \mathrm{MeV}$ to $\geq 100 \mathrm{MeV}$ (Benz 2008) with only convective electric fields. Parallel electric fields appear to be necessary for further higher energy accelerations.

To include a parallel electric field consistently in a MHD model, the existence of a switched on anomalous resistivity is necessary. Therefore a reliable microscopic model is a key factor to generate resistive electric fields and accelerating particles adiabatically to the observed energies. Microphysical kinetic descriptions, which provide the resistivity expressions for the macroscopic MHD coefficients (Büchner \& Elkina 2006), are necessarily required for this purpose. Our MHD code, although tried to include the resistive electric fields, has failed to run long enough for the resistivity to have been switched on in coronal regions where the particles are considered to be initially accelerated. Besides, the parallel electric fields obtained from kinetic processes are in general confined to regions on the ion inertia scale, which is much smaller than the macroscopic MHD grid scales. A multi-scale MHD simulation with a much higher resolution at the dissipation region can somehow decrease this scale gap. More work needs to be done on for longer MHD simulations with higher resolution to generate parallel resistive electric fields to study adiabatic (direct) acceleration.
Besides, the present paper is limited to investigate the mechanisms and processes of acceleration by a null point instead of giving an indication of how many particles could reach the null and get the chance to gain energy. Future work will be focusing on the probability of particles reaching the null point and being accelerated. Moreover, the accelerated spectrum of a large amount of particles with an initial Maxwellian distribution will also be studied and compared with data, in particular from RHESSI.

Acknowledgements. The authors are very grateful to the referee for helpful comments and would like to thank Lyndsay Fletcher, Anna Lisa Restante and Miroslav Barta for many fruitful discussions. The work by J.-N. Guo, J. Büchner, and E. Marsch is supported by the European Community Research Training Network project SOLAIRE under the project number 35484. The work of W.-Q. Gan is supported by NNSFC (10773031 and 10833007) and MSTC (2006CB806302).

\section{Appendix A: Initial configuration of the magnetic field}

We used a four mode Fourier expansion for the potential extrapolation of the magnetic field. We considered a square geometry ( $L_{x}=L_{y}=L$ ) and line symmetry condition at four side boundaries. The resulting 3D quadrupolar magnetic field has the following analytical solutions

$$
\begin{aligned}
& B_{x}=\exp \left(-\lambda_{2} z\right)\left[25 \sin \frac{2 \pi}{L} x\right]+\exp \left(-\lambda_{4} z\right)\left[-125 \sin \frac{4 \pi}{L} x\right] \\
& +\frac{\exp \left(-\lambda_{22} z\right)}{\sqrt{2}}\left[-75 \cos \frac{2 \pi}{L} x \sin \frac{2 \pi}{L} y+75 \sin \frac{2 \pi}{L} x \cos \frac{2 \pi}{L} y\right] \\
& B_{y}=\exp \left(-\lambda_{2} z\right)\left[25 \sin \frac{2 \pi}{L} y\right]+\exp \left(-\lambda_{4} z\right)\left[125 \sin \frac{4 \pi}{L} y\right] \\
& \quad+\frac{\exp \left(-\lambda_{22} z\right)}{\sqrt{2}}\left[-75 \sin \frac{2 \pi}{L} x \cos \frac{2 \pi}{L} y+75 \cos \frac{2 \pi}{L} x \sin \frac{2 \pi}{L} y\right] \\
& B_{z}=\exp \left(-\lambda_{2} z\right)\left[25 \cos \frac{2 \pi}{L} y+25 \cos \frac{2 \pi}{L} x\right] \\
& \quad+\exp \left(-\lambda_{4} z\right)\left[125 \cos \frac{4 \pi}{L} y-125 \cos \frac{4 \pi}{L} x\right] \\
& +\exp \left(-\lambda_{22} z\right)\left[75 \sin \frac{2 \pi}{L} x \sin \frac{2 \pi}{L} y+75 \cos \frac{2 \pi}{L} x \cos \frac{2 \pi}{L} y\right]
\end{aligned}
$$

\section{References}

Aschwanden, M. 2002, Space Sci. Rev., 101, 1

Aschwanden, M., Kosugi, T., Hudson, H., Wills, M., \& Schwartz, R. 1996, ApJ, 470, 1198

Aulanier, G., DeLuca, E., Antiochos, S., McMullen, R., \& Golub, L. 2000, ApJ, 540,1126

Benz, A. 2008, Living Rev. Sol. Phys., 5

Brown, J. 1971, Sol. Phys., 18, 489

Browning, P., \& Vekstein, G. 2001, J. Geophys. Res., 106, 18677 Bruhwiler, D., \& Zweibel, E. 1992, J. Geophys. Res., 97, 10825

Büchner, J. 1986, Astron. Nachr., 307, 191

Büchner, J. 2006, Space Sci. Rev., 122, 149

Büchner, J. 2007, in New Solar Physics with Solar-B Mission, ed. K. Shibata, S. Nagata, \& T. Sakurai, ASP, 369, 407

Büchner, J., \& Elkina, N. 2006, Space Sci. Rev., 121, 237

Büchner, J., \& Zelenyi, L. M. 1989, J. Geophys. Res., 93, 11.821

Büchner, J., Nikutowski, B., \& Otto, A. 2004, in Proceedings of the SOHO 15 Workshop-Coronal Heating, 6-9 September 2004, St. Andrews, Scotland, UK (ESA SP-575), ed. R. W. Walsh, J. Ireland, D. Danesy, \& B. Fleck (Paris: European Space Agency), 23 
J.-N. Guo et al.: Is the 3-D magnetic null point with a convective electric field an efficient particle accelerator?

Craig, I., \& Litvinenko, Y. 2002, ApJ, 570, 387

Dalla, S., \& Browning, P. 2005, A\&A, 436, 1103

Dalla, S., \& Browning, P. 2008, A\&A, 491, 289

Des Jardins, A., Canfield, R., Longcope, D., Fordyce, C., \& Waitukaitis, S. 2009, ApJ, 693, 1628

Dmitruk, P., Matthaeus, W., Seenu, N., \& Brown, M. 2003, ApJ, 597, 81

Efthymiopoulos, C., Gontikakis, C., \& Anastasiadis, A. 2005, A\&A, 443, 663

Fletcher, L., Metcalf, T., Alexander, D., Brown, D., \& Ryder, L. 2001, ApJ, 554, 451

Hamilton, B., Fletcher, L., McClements, K., \& Thyagaraja, A. 2005, ApJ, 625, 496

Hannah, I., \& Fletcher, L. 2006, Sol. Phys., 236, 59

Heerikhuisen, J., Litvinenko, Y., \& Craig, I. 2002, ApJ, 566, 512

Hesse, M., Schindler, K., Birn, J., \& Kuznetsova, M. 1999, Physics of Plasmas, 6,1781

Karlicky, M., \& Barta, M. 2006, ApJ, 647, 1472

Lau, Y., \& Finn, J. 1990, ApJ, 350, 672

Lin, R., \& Hudson, H. 1976, Sol. Phys., 50, 153

Litvinenko, Y. 1996, ApJ, 462, 997
Liu, W., Chen, P., Ding, M., \& Fang, C. 2009, ApJ, 690, 1633

Masuda, S., Kosugi, T., Hara, H., Tsuneta, S., \& Ogawara, Y. 1994, Nature, 371, 495

Mori, K., Sakai, J., \& Zhao, J. 1998, ApJ, 494, 430

Northrop, T. 1963, Rev. Geophys, 1, 283

Otto, A., Büchner, J., \& Nikutowski, B. 2007, A\&A, 468, 313

Potter, D. 1973, Computational Physics (John Wiley \& Sons)

Priest, E., \& Titov, V. 1996, Phil. Trans. R. Soc. Lond. A, 354, 2951

Restante, A., Aulanier, G., \& Parnell, C. 2009, A\&A, 508, 433

Sakai, J. 1992, Sol. Phys., 140, 99

Santos, J., \& Büchner, J. 2007, Astrophys. Space Sci. Trans., 3, 29

Schopper, R., Birk, G., \& Lesch, H. 1999, Phys. Plas., 6, 4318

Speiser, T. 1965, J. Geophys. Res., 70, 4219

Speiser, T. 1967, J. Geophys. Res., 72, 3919

Turkmani, R., Cargill, P., Galsgaard, K., Vlahos, L., \& Isliker, H. 2006, A\&A, 449, 749

Vekstein, G., \& Browning, P. 1997, Phys. Plasmas, 4, 2261

Wood, P., \& Neukirch, T. 2005, Sol. Phys., 226, 73

Zharkova, V., \& Gordovskyy, M. 2004, ApJ, 604, 884 